\title{
Cultural Complications Curriculum: Applicability to Surgical Oncology Programs and Practices
}

\author{
Alison S. Baskin, BA ${ }^{1}$, Lesly A. Dossett, MD, MPH, FACS ${ }^{2}$, and Chelsea A. Harris, MD, MS \\ ${ }^{1}$ University of Michigan School of Medicine, Ann Arbor, MI; ${ }^{2}$ Department of Surgery, University of Michigan, Ann Arbor, \\ MI; ${ }^{3}$ Department of Surgery, University of Maryland Medical Center, Baltimore, MD
}

\begin{abstract}
Background. Recognizing the need to raise awareness of core diversity, equity, and inclusion (DEI) issues in the healthcare system, our previously developed Cultural Complications Curriculum aims to support institutions in reducing cultural error. As we continue program deployment, we discuss the opportunity to apply the Cultural Complications Curriculum to multidisciplinary audiences, such as in cancer programs.
\end{abstract}

Methods. We discuss applicability of the Cultural Complications Curriculum to cancer programs and practices, including how to tailor case discussions to oncology audiences. By emphasizing the unique characteristics of the multidisciplinary care environment and anticipating potential barriers to curriculum implementation, we demonstrate how the Cultural Complications Curriculum may support culture improvement across broad audiences. Results. The successful application of the Cultural Complication Curriculum to multidisciplinary care programs will depend on appreciating differences in background knowledge, tailoring discussions to audience needs, and adapting material by incorporating new data and addressing emerging DEI issues. Multidisciplinary environments may require innovative approaches to education including virtual platforms, increased collaboration across centers and systems, and support from professional societies. In integrated care environments, like oncology, effective DEI

(C) Society of Surgical Oncology 2021

First Received: 26 April 2021

Accepted: 17 May 2021;

Published Online: 9 June 2021

L. A. Dossett, MD, MPH, FACS

e-mail: 1dossett@med.umich.edu; ldossett@umich.edu discussions call for the engagement of a variety of medical specialties and departments.

Conclusions. To meet the needs of an increasingly diverse patient population and workforce, our approach to DEI education must embrace the interdependent nature of care delivery. In oncology and other multidisciplinary care environments, application of the Cultural Complications Curriculum may be the first step to combating cultural error by engaging a broader demographic within our healthcare system.

The COVID-19 pandemic, numerous examples of cancer and healthcare disparities, and ongoing surges in police brutality and domestic hate crimes have most recently heighted our need to fight bias, discrimination, and harassment. Many institutions have responded to these national crises by intensifying antiracism efforts within their own walls by assembling diversity, equity, and inclusion (DEI) offices, advisory boards, and quality-improvement frameworks. ${ }^{1}$ At the same time, a number of organizations including the Society for Surgical Oncology (SSO) have released position statements explicitly condemning racism and highlighting missions to support individuals regardless of their race, ethnicity, gender, sexual orientation, or socioeconomic status. ${ }^{2}$ Individuals and communities have engaged through social media platforms and virtual panels discussing inequities within both healthcare and greater society. ${ }^{3}$ While these efforts seemingly demonstrate an increased enthusiasm for diversity and condemnation of discrimination, as time has repeatedly shown, an avowal of advocacy, regardless of how well intentioned it may be, is not enough to effect long-term behavioral change. 
Recognizing the need to systematically transform culture at our own institutions and noting multiple deficiencies within standard bias training, we developed and implemented a "Cultural Complications" program (www.cultura lcomplications.com) aimed at supporting departments wishing to address cultural error. ${ }^{4,5}$ The Cultural Complications Curriculum leverages the traditional morbidity \& mortality $(\mathrm{M} \& \mathrm{M})$ conference structure, featuring case presentations, didactic sessions, and group discussions in a regular, dedicated monthly forum intended to raise awareness of core DEI issues in the hospital. Integration of the Cultural Complications Curriculum into a preexisting and standing conference (like $\mathrm{M} \& \mathrm{M}$ ) demonstrates a commitment to culture change from departmental leadership and promotes a learning platform where faculty and trainees are engaged simultaneously. ${ }^{5}$

As we have gained experience in Cultural Complications at our own institutions through supporting the efforts of others who have gained familiarity with this program and/or launched similar initiatives, we have recognized how this program may be uniquely applicable to the multidisciplinary audiences the exist in cancer programs. In these settings where a variety of perspectives exist, we believe Cultural Complications is well suited to facilitate culture change by establishing a common knowledge base among program participants, improving communication surrounding DEI issues, and supporting strategy development to combat bias, harassment, and discrimination in dynamic environments. Notably, there is an opportunity to target cultural error occurring in oncology, where a multidisciplinary approach is best practice for the delivery of patient care. Here, we discuss the applicability of the Cultural Complications Curriculum to oncology audiences and how it can be tailored for multidisciplinary settings to inform discussions across a range of individuals and support ongoing and future efforts to eliminate cultural error in our medical community.

\section{DEFINING THE SCOPE OF BIAS AND DISPARITIES IN ONCOLOGY}

It has been well documented that minority populations face a disproportionate cancer burden due to factors of structural racism. Patients from minority backgrounds are more likely to encounter barriers accessing healthcare services, such as cancer screenings, for reasons that are likely multifactorial and due in part to disparities in insurance status and/or ability to attend medical appointments, differential perceptions of personal risk, or decreased trust in the healthcare system. ${ }^{6-8}$ Limited healthcare access may result in a later disease presentation and contribute to the lower rates of surgical resection seen in minority groups. ${ }^{9}$ Surgical decision-making also has been shown to vary based on a patient's background and may result in screening or treatment recommendations diverging from the standard of care. Furthermore, insufficient clinical and genetics research on diverse populations leads to poor recognition of clinical considerations that may uniquely exist for minorities and therefore reduced generalizability of findings and less appropriate or tailored treatment guidelines. ${ }^{10}$ Taken altogether, patients from minority groups are more likely to have worse cancer outcomes, demonstrating the direct clinical impact of racism and bias in surgery and medicine. ${ }^{11}$

Similarly, harassment and discrimination in the workplace is an ongoing insult to healthcare professionals and contributes to the lack of diversity within the surgical oncology workforce. ${ }^{12}$ Failure to foster and invest in a pipeline of surgical and medical trainees from diverse backgrounds perpetuates underrepresentation of minority physicians. Furthermore, because social and professional networks often play a role in determining inclusion in professional societies, clinicians who have not historically had the same social capital and privilege as others, are disadvantaged from joining these groups. ${ }^{13}$ In the same way, physicians from minority backgrounds may be passed over for promotions or leadership roles. Lectures and educational panels without a diverse array of speakers send a message that the voices of women and minority groups are not valued. ${ }^{14}$ The downstream effects of these biases, in combination with the overt acts of harassment that too frequently take place within the workplace, are devastating to both physicians and patients. Given the emotional and physical tolls and high stakes of both providing and receiving cancer care, we have an outstanding duty to address cultural transgressions in our workplaces.

While the Cultural Complications Curriculum alone will not solve the deep-seated issues of structural racism, we hope that by highlighting issues of bias through increased dialogue within medical communities, this program will empower individuals to become changemakers at their own institutions by providing them the knowledge base and language to provoke further discussion and take meaningful action. Furthermore, in multidisciplinary settings, such as cancer centers, this curriculum may be especially well positioned to promote conversations that elevate different perspectives, which may otherwise remain unheard, and work toward developing strategies that fight core DEI issues by bringing together health care professionals from varying backgrounds, clinical practices, and training levels. 


\section{MULTIDISCIPLINARY APPLICATION OF THE CULTURAL COMPLICATIONS CURRICULUM}

\section{An Opportunity in Cancer Care}

The Cultural Complications Curriculum aims to educate learners at all levels and elicit an array of perspectives. Multidisciplinary cancer care teams are comprised of a variety of physicians, nurses, and other health care professionals, which inherently involve different viewpoints, making application of the Cultural Complications Curriculum to the oncology setting naturally fitting. ${ }^{15}$ In cancer care, our roles as providers are so interdependent that if one member of the interdisciplinary team does not meet the cultural needs of a patient or fellow provider, then the efforts of others to do so will fail. The entwined nature of cancer treatment and management emphasizes a need to educate and hold accountable one another for personal and collective biases. We should aim to unite health care professionals against bias, harassment, and discrimination in the same way that we routinely align ourselves to improve patient outcomes.

\section{Determining and Targeting an Audience in Oncology}

When applying the Cultural Complications Curriculum to a multidisciplinary setting, it is important to know and understand your audience, particularly in regards to their cultural knowledge gaps and willingness to welcome change. This means assessing the breadth of professional and training levels and the specialties of audience members, recognizing there may be a greater variety of participant needs than if the audience all belonged to a single department. When applying the current program to a targeted clinical audience in the multidisciplinary setting, participants' background knowledge may vary substantially. Therefore, it is especially important to provide adequate context and background information to set the stage for discussion, build a shared knowledge base, and familiarize a diverse team with a common language, while recognizing that a key to increasing cultural competency in the academic setting is through a data-driven process.

Furthermore, to reinforce that issues of bias, harassment, and discrimination occur in the audience's own environment and maximize buy-in from audience members, we recommend highlighting local issues to supplement the preexisting Cultural Complications case bank. One potential approach to identifying issues tailored to specific audiences is to utilize anonymous surveys where respondents can submit cases they have encountered in their respective workplaces. Submissions can be modified to retain anonymity, while remaining authentic to the raised issue. Solicitation of issues from participants may also help to highlight the diversity of experiences in a multidisciplinary setting and uncover new blind spots for some members of the multidisciplinary team.

With growing bodies of research and new treatment approaches to cancer, the field of oncology is constantly evolving. As a result, champions of the Cultural Complications Curriculum must be especially willing to adapt conversations and cases to feature new data and shed light on arising DEI issues. We have demonstrated how the current Cultural Complications Curriculum may be applied to oncology-geared audiences, discussing the original DEI themes from our program using oncology-related examples (Table 1). Background data from our preexisting modules may also be replaced or supplemented to best suit targeted audiences.

\section{Removing Barriers to Implementing the Curriculum}

One concern when applying the Cultural Complications Curriculum to the multidisciplinary setting may be the absence of built-in M\&M conferences. However, at institutions where departmental M\&M sessions are not standing meetings or multidisciplinary $M \& M$ sessions may not exist, there are opportunities to develop alternative forums for discussion. For example, a virtual "Diversity Tumor Board" recently sponsored by the SSO highlighted cases of structural racism (e.g. screening guidelines for colorectal cancer) and disparities in transgender cancer care (e.g. differential access to care), bringing together clinicians from several institutions with different backgrounds and clinical interests in oncology. ${ }^{3}$ This multidisciplinary initiative demonstrated that cultural error can be discussed effectively in innovative environments with the same rigor as in a traditional conference setting. As we continue to fight the COVID-19 pandemic, which has transformed the workplace in many ways, including accelerating the adoption of digital learning and patient care, institutions may continue to leverage virtual platforms to coordinate conferences on DEI issues in a multidisciplinary space. Many virtual platforms offer the ability to anonymously survey audience members regarding their experiences, as well as the option to record the session, which can be used to build a library of these presentations for future learning and reference.

Additionally, some departments or multidisciplinary centers may feel they lack the expertise to host discussions on DEI topics, which can discourage them from launching the Cultural Complications Curriculum or similar initiatives at their own institution. Furthermore, they may not have university-based resources (e.g., funding, time, formalized leadership) necessary to facilitate these efforts. To address this potential barrier, institutions may consider 
TABLE 1 Selected examples of cultural complications curriculum themes adapted for oncology

\begin{tabular}{|c|c|}
\hline Cultural complication theme & Oncology case example \\
\hline Business case for diversity & Lack of diversity of in surgical oncology workforce and barriers to promotion for underrepresented physicians. \\
\hline $\begin{array}{l}\text { Leadership and national } \\
\text { representation }\end{array}$ & $\begin{array}{l}\text { Minority patients are less likely to be enrolled in clinical trials studying cancer, leading to guidelines that do not } \\
\text { reflect differential genetic risk. }\end{array}$ \\
\hline $\begin{array}{l}\text { Vigilance and } \\
\text { intersectionality }\end{array}$ & $\begin{array}{l}\text { Failing to recognize the challenge of traveling to daily adjuvant radiation appointments faced by patient from } \\
\text { lower SES. }\end{array}$ \\
\hline $\begin{array}{l}\text { Implicit bias and } \\
\text { microaggression }\end{array}$ & $\begin{array}{l}\text { Placing blame or making snide remark about patient's extensive smoking history when diagnosed with lung } \\
\text { cancer. }\end{array}$ \\
\hline Stereotype threat & $\begin{array}{l}\text { Marginalized patient has a delayed presentation of cancer and provider blames patient for not taking better care } \\
\text { of self. }\end{array}$ \\
\hline Mentoring and sponsorship & $\begin{array}{l}\text { A transgender patient wants to discuss continuing their hormone therapy during breast cancer treatment with an } \\
\text { LGBTQ+ provider but is unsure how to identify one. }\end{array}$ \\
\hline
\end{tabular}

This table demonstrates how the Cultural Complications Curriculum can be adapted for an oncology-oriented audience. Potential sample cases for 6 of the 12 program themes are provided here

SES socioeconomic status

collaborating across departments, centers, or systems to unify resources, recruit known champions for support, and invite DEI content experts to help launch initiatives in a way that is mutually beneficial for all parties. Professional societies, such as SSO, may consider developing a directory of DEI content experts, from which centers lacking their own experts may draw on for guidance.

\section{Sustainability, Partnership, and Future Direction}

Application of the Cultural Complications Curriculum to the multidisciplinary setting offers an opportunity to raise awareness of core DEI issues beyond a single department to reach a broader medical community providing interconnected patient care. Doing so may highlight a greater breadth of cultural error issues, elicit different perspectives, and offer richer discussion. Specifically, at multidisciplinary cancer centers, there are opportunities to include a variety medical specialties (e.g., surgical oncology, medical oncology, radiation oncology) and departments in DEI discussions, which can be particularly valuable given that members from these different clinical homes often concurrently play a role in single patient's treatment course. Furthermore, in the multidisciplinary setting, we encourage centers to invite other individuals who are involved in patient care or otherwise part of the local workforce (e.g., patient advocates, nurses, hospital administration) to participate.

For many institutions, implementation of the Cultural Complications Curriculum may be the first step in changing culture-providing a learning platform to raise awareness, share knowledge, and promote discussion of ongoing bias, harassment, and discrimination. While recognizing and commending institutions' efforts to better understand how cultural error occurs within their own walls, the work to improve culture in patient care and the workplace does not end here. Instead, we must continue to identify how we can translate learned lessons into actionable policies and build systems that reduce structural racism, discrimination, and harassment. Specifically within oncology, it will be critical to meet the needs of an increasingly culturally and racially diverse patient population and support and recruit a workforce that better represents all minority groups.

FUNDING Dossett is supported by a grant from the Agency for Healthcare Research and Quality (AHRQ; 5 K08 HS026030-02). Alison Baskin receives scholarship funding from the University of Michigan Rogel Cancer Center.

\section{REFERENCES}

1. Rotenstein LS, Reede JY, Jena AB. Addressing workforce diversity: a quality-improvement framework. $N$ Engl J Med. 2021;384(12):1083-6.

2. Society of Surgical Oncology. Society of Surgical Oncology Statement on Racism, Diversity and Cancer Care. 2020. https:// www.surgonc.org/about-sso/society-of-surgical-oncology-statem ent-on-racism-diversity-and-cancer-care/. Accessed 26 Mar 2021.

3. Society of Surgical Oncology. SSO Virtual Tumor Board: A Case-Based Discussion on Treating Diverse Populations. 2020. h ttps://experted.surgonc.org/Public/Catalog/Details.aspx?id=aMu RIN4\%2BKjs\%2BZVENLkohnQ\%3D\%3D\&returnurl=\%2FUser s\%2FUserOnlineCourse.aspx\%3FLearningActivityID\%3DaMuR $1 \mathrm{~N} 4 \% 252 \mathrm{bKjs} \% 252 \mathrm{bZVENLkohnQ} \% 253 \mathrm{~d} \% 253 \mathrm{~d}$. Accessed 24 Feb 2021.

4. Harris CA, Dimick JB, Dossett LA. Cultural complications: a novel strategy to build a more inclusive culture. Ann Surg. 2021;273(3):e97-99. https://doi.org/10.1097/SLA.00000000000 04219.

5. Harris CA, Dimick JB, Dossett LA. Cultural complications: why, how, and lessons learned. Am J Surg. 2021;221(3):609-11. h ttps://doi.org/10.1016/j.amjsurg.2020.09.002. 
6. Adams LB, Richmond J, Corbie-Smith G, Powell W. Medical mistrust and colorectal cancer screening among African Americans. J Community Health. 2017;42(5):1044-61.

7. Greenberg CC, Weeks JC, Stain SC. Disparities in oncologic surgery. World J Surg. 2008;32(4):522-8.

8. Gordon HS, Street RL Jr, Sharf BF, Kelly PA, Souchek J. Racial differences in trust and lung cancer patients' perceptions of physician communication. J Clin Oncol. 2006;24(6):904-9.

9. Farjah F, Wood DE, Yanez ND 3rd, et al. Racial disparities among patients with lung cancer who were recommended operative therapy. Arch Surg. 2009;144(1):14-8.

10. Hamel LM, Penner LA, Albrecht TL, Heath E, Gwede CK, Eggly S. Barriers to clinical trial enrollment in racial and ethnic minority patients with cancer. Cancer Control. 2016;23(4):327-37.

11. Lam MB, Raphael K, Mehtsun WT, et al. Changes in racial disparities in mortality after cancer surgery in the US 2007-2016. JAMA Netw Open. 2020;3(12):e2027415.
12. Newman LA, Pollock RE, Johnson-Thompson MC. Increasing the pool of academically oriented African-American medical and surgical oncologists. Cancer. 2003;97(1 Suppl):329-34.

13. Yehia BR, Cronholm PF, Wilson N, et al. Mentorship and pursuit of academic medicine careers: a mixed methods study of residents from diverse backgrounds. BMC Med Educ. 2014;14:26.

14. Collins FS. Time to end the manel tradition. 2019. https://www. nih.gov/about-nih/who-we-are/nih-director/statements/time-endmanel-tradition. Accessed 26 Mar 2021.

15. Selby P, Popescu R, Lawler M, Butcher H, Costa A. The value and future developments of multidisciplinary team cancer care. Am Soc Clin Oncol Edu Book. 2019;39:332-40.

Publisher's Note Springer Nature remains neutral with regard to jurisdictional claims in published maps and institutional affiliations. 